\title{
Comparison of dietary intakes and anthropometric measurements among Native Filipino Nurses and Immigrant Filipino Nurses in the UK - a potential role for acculturation?
}

Acculturation, including dietary acculturation, is commonly observed in immigrant populations and may constitute an independent risk factor for health outcomes ${ }^{(1)}$. Changes in dietary patterns and an increased incidence of obesity compared to native Filipinos have been reported in Filipino immigrant populations in the US ${ }^{(2)}$. In the UK, Filipino immigrant nurses make up $91 \%$ of the UK nursing workforce $^{(3)}$, yet to date little is known about their dietary patterns and the effect of acculturation on this population group.

The aims of this study were 1) to compare dietary intakes and anthropometric measurements of native Filipino nurses in the Philippines (PH) and first-generation immigrant Filipino nurses in the UK and 2) to investigate the level of acculturation, effect of dietary acculturation and changes in anthropometric measurements among UK cohorts.

Healthy male and female nurses aged 25-60 were recruited via direct contacts and by word of mouth from three hospitals in the Philippines and three in the UK (April to June 2018). Information was collected for anthropometric measurements, physical activity levels and $24 \mathrm{~h}$ dietary recalls, and participants completed a sociodemographic questionnaire and A Short Acculturation Scale for Filipino British Questionnaire and Dietary Acculturation Questionnaires.

The study sample consisted of 39 native Filipino nurses and 36 immigrant Filipino nurses residing in the UK $(n=75)$. There was no significant difference between the total energy intake, the relative contribution of macronutrients to daily dietary intake and PAL levels of the two groups $(\mathrm{p}>0.05)$ and a strong negative correlation was observed between BMI and PAL in both cohorts (PH: $\mathrm{r}=-.935$; UK: $\mathrm{r}=-.758, p<0.01)$. After adjusting for age, BMI was significantly higher among UK female nurses compared to PH female nurses (UK, 26.51, PH, 22.48, $p<0.05$ ). The acculturation scores indicated the emerging biculturalism among Filipino immigrants in the UK (ASASFB= 2.76). Dietary acculturation scores suggested the maintenance of traditional Filipino eating patterns and a moderate adaptation of Western dietary patterns in the UK cohort (DAQFBf $=3.80$, DAQFBw $=6.39)$. Filipino dietary acculturation was weakly, but significantly and negatively correlated with weight $(\mathrm{r}=-.347, p<0.05)$, indicating that those who maintained a traditional Filipino dietary pattern potentially had a lower incidence of being overweight and obese.

This research study provides an insight on dietary pattern and anthropometric characteristics of first-generation Filipino immigrants in the UK with potential implications for their overall health status.

1. Lee J, Brancati F \& Yeh H (2011). Diabetes Care 34, 353-357.

2. Araneta M, Lantion-Ang L, Grandinetti A et al. (2006). Diab Res and Clin Prac 71, 306-312.

3. House of Commons Health Committee (2018). The Nursing Workforce 2017-19. 